\title{
Virulence et résistance : deux caractéristiques antagonistes chez Escherichia coli?
}

\author{
Virulence and resistance: two antagonistic properties in Escherichia coli ?
}

\author{
E. Denamur - B. Picard \\ C SRLF et Springer-Verlag France 2012
}

\begin{abstract}
À l'image de la notion d'espèces généralistes et spécialistes chez les animaux, les bactéries sont-elles aptes à être à la fois virulentes et résistantes, ou se spécialisent-elles dans une de ces deux propriétés ? Pour répondre à cette question, nous prendrons l'exemple de l'espèce Escherichia coli dont les représentants ont des modes de vie variés. En effet, E. coli se partage entre son habitat primaire, le tube digestif des vertébrés, dont l'homme, et son environnement secondaire, l'eau et les sédiments [1]. De plus, E. coli est un redoutable pathogène, responsable d'environ deux millions de morts chaque année chez l'homme en causant divers types de diarrhées et d'infections extra-intestinales, telles que les infections urinaires, les méningites chez le nouveau-né et les bactériémies [2]. Dans cette espèce à structure de population globalement clonale, les souches se répartissent en quatre groupes phylogénétiques majeurs nommés $\mathrm{A}, \mathrm{B} 1, \mathrm{~B} 2$ et $\mathrm{D}$ [3]. Il a été rapporté un lien entre la virulence extra-intestinale et la phylogénie, les souches des groupes $\mathrm{B} 2$ et $\mathrm{D}$ étant retrouvées majoritairement dans les pathologies extra-intestinales : elles sont en effet porteuses de nombreux gènes de virulence type adhésines, toxines, systèmes de capture du fer et capsules [4]. À l'inverse, les souches retrouvées à l'état commensal dans le tube digestif de sujets sains sont plutôt des groupes phylogénétiques A et B1 et sont dépourvues de gène de virulence [5]. Dans ce contexte, comment se situe la résistance aux antibiotiques?
\end{abstract}

\section{Les données épidémiologiques}

C'est au début des années 1990 qu'il a été rapporté pour la première fois que dans une collection de 75 souches de septi-

\footnotetext{
E. Denamur $(\bowtie)$

INSERM U722, Faculté de médecine de Paris Diderot, site Xavier Bichat, 16, rue Henri Huchard, F-75018 Paris, France e-mail : erick.denamur@inserm.fr

B. Picard

UMR 722 INSERM et Université Paris Nord,

PRES Sorbonne Paris Cité
}

cémie à point de départ urinaire, les souches qui possèdent le plus de facteurs de virulence appartiennent au groupe phylogénétique $\mathrm{B} 2$ mais présentent de manière statistiquement significative moins de résistance aux antibiotiques [6]. Ceci était montré pour le score de résistance global ou par antibiotique. Les antibiotiques concernés étaient les bétalactamines, les aminosides, les cyclines et les sulfamides. Cette relation inverse entre virulence et résistance a ensuite été retrouvée dans de nombreux endroits du monde sur des collections variées de souches et continue d'être observée dans des études récentes portant sur des centaines de souches [7-9]. Une littérature particulièrement abondante existe sur la notion de souches résistantes aux quinolones qui possèdent peu de facteurs de virulence et appartiennent rarement au groupe phylogénétique B2 [10,11]. Les intégrons, des boîtes à outils moléculaires permettant la capture et l'expression des gènes de virulence, ont également été retrouvés moins fréquemment chez les souches du groupe phylogénétique B2 [12].

\section{Le substratum moléculaire}

Au-delà de ces constatations épidémiologiques, quel peut être le mécanisme moléculaire expliquant cet antagonisme apparent entre virulence et résistance ? Chez $E$. coli, la virulence extra-intestinale est liée à la présence des facteurs de virulence cités plus haut qui sont le plus souvent regroupés sur des fragments d'ADN génomique appelés « îlots de pathogénicité » [13]. Il a été clairement montré que la virulence intrinsèque des souches, mesurée dans un modèle murin de septicémie, était proportionnelle au nombre de facteurs de virulence $[4,14,15]$, et par conséquence au nombre d'îlots de pathogénicité [16].

Des auteurs ont étudié la capacité des quinolones à induire la perte de facteurs de virulence tels que l'hémolysine, le facteur nécrosant cytotoxique 1, l'adhésine $\mathrm{P}$ fimbriae et l'autotransporteur Sat chez trois souches d'E. coli responsables d'infection urinaire et appartenant au groupe phylogénétique B2 [17]. Ces facteurs de virulence se trouvaient sur des îlots 
de pathogénicité. En faisant une sélection de la résistance à la ciprofloxacine en plusieurs étapes en incubant les souches avec des concentrations subinhibitrices d'antibiotiques, les auteurs ont vu une perte partielle ou totale de l'îlot de pathogénicité contenant l'hémolysine et le facteur nécrosant. Comme les quinolones induisent le système SOS chez les bactéries, les auteurs ont étudié si les souches mutantes pour la protéine RecA, la protéine majeure du système SOS, perdaient aussi leurs gènes de virulence en présence de ces antibiotiques. Ils ont montré que l'inactivation de RecA n'affectait que la perte partielle de l'îlot de pathogénicité induit par les quinolones. Ils ont également vérifié qu'il n'y avait pas de perte spontanée de l'îlot de pathogénicité lorsque les souches étaient incubées en l'absence de quinolones. Les auteurs ont donc conclu que les quinolones induisaient une perte partielle ou totale des îlots de pathogénicité in vitro chez des souches uropathogènes d'E. coli du groupe phylogénétique B2 par des voies respectivement SOS-dépendantes ou indépendantes [17]. Ces résultats n'ont toutefois pas été confirmés par d'autres auteurs sur un panel différent de souches [18].

Le séquençage de génomes complets de bactéries a aussi ouvert des pistes sur les mécanismes moléculaires de ce compromis entre résistance et virulence. Le génome des souches d'E. coli est très dynamique avec de très nombreux gains et pertes de gènes au cours de l'évolution de la bactérie [19]. Ainsi, alors qu'un génome d'E. coli est composé d'environ 4500 gènes, moins de 2000 sont communs à l'ensemble des souches de l'espèce, révélant un répertoire de plusieurs dizaines de milliers de gènes pour l'espèce. Toutefois, ces gains et pertes de gènes se font à des endroits précis sur le chromosome, des « points chauds d'insertion/ délétion », qui ne perturbent pas la physiologie de la bactérie. C'est ce qui a fait dire qu'il y avait dans les génomes bactériens « de l'ordre dans le désordre » [20]. De plus, l'organisation de ces segments gagnés ou perdus et qui comprennent les îlots de pathogénicité cités plus haut est modulaire, c'est-à-dire que l'on retrouve chez les différentes souches certains fragments de ces îlots en plus ou moins grand nombre [19]. Le séquençage complet d'une souche d'E. coli d'un clone émergent résistant à l'ampicilline, chloramphenicol, streptomycine, sulfamides, tétracycline et trimethoprime a montré que la majorité des gènes codant pour cette résistance étaient localisés dans une région chromosomique de 23 kilobases [21]. Ce module génomique de résistance était lui-même situé dans un îlot génomique de 105 kilobases localisé à l'ARNt leucine. Le module génomique de résistance était caractérisé par de nombreux restes d'événements de réarrangement et de mobilisation suggérant de multiples transferts horizontaux de gènes. En comparant cette région de l'ARNt leucine chez d'autres souches séquencées d'E. coli, il a été montré que cette région était un point chaud d'intégration et que l'on pouvait avoir alter- nativement selon les souches à cet endroit soit un îlot de résistance, soit un îlot de virulence [21].

\section{Qu'en est-il avec l'avènement des bêtalactamases à spectre élargi et des carbapénémases ?}

Le niveau de résistance des souches de l'espèce $E$. coli s'est considérablement modifié avec l'apparition dans les années 2000 de souches productrices de bêta-lactamases à spectre élargi de type TEM, SHV et surtout CTX-M rendant les souches résistantes aux céphalosporines de troisième génération [22]. Plus récemment, des souches productrices de carbapénèmases et inactivant les pénèmes ont été rapportées [23]. Ces souches sont de plus en plus souvent résistantes aux antibiotiques des autres familles. Ce phénomène épidémique explosif a des origines moléculaires complexes avec diffusion de clones bactériens, de plasmides portant la résistance et des gènes de résistance eux-mêmes qui se trouvent sur des séquences mobiles [24]. Un des clones diffusant de façon planétaire les bêtalactamases à spectre étendu appartient au groupe phylogénétique B2 et correspond au séquençotype 131 [25]. Ce clone, bien que possédant relativement peu de facteurs de virulence classique, est virulent dans un modèle de septicémie chez la souris [26]. Ce même clone vient d'être rapporté, produisant la carbapénèmase NDM-1 [27]. Ainsi, on peut constater qu'avec la pression de sélection due aux antibiotiques s'accentuant, des souches ayant à la fois des capacités de virulence et de résistance apparaissent.

\section{Conclusions}

Il est incontestable que les bactéries ont une tendance à être des spécialistes et qu'elles sont plutôt, soit virulentes, soit résistantes. Les mécanismes moléculaires responsables de ce compromis sont mal élucidés et restent à déterminer. Toutefois, avec l'émergence des souches productrices de bêtalactamases à spectre élargi et de carbapénèmases suite à l'augmentation de la pression de sélection antibiotique, il est clair que l'on peut isoler des bactéries généralistes qui sont tout à la fois virulentes et résistantes.

Ces données sur le compromis résistance-virulence sont aussi à interpréter dans la complexité du processus infectieux chez l'homme qui survient sur un terrain variable plus ou moins sensible à l'infection pour des raisons génétiques ou acquises d'immunodépression générale ou locale. Dans ce contexte, des bactéries plus ou moins virulentes peuvent entrainer une infection [28] et la gravité d'une infection n'est pas corrélée à la présence de facteurs de virulence ou à la virulence intrinsèque de la souche mesurée dans un modèle animal [29]. 
Conflit d'intérêt : les auteurs déclarent ne pas avoir de conflit d'intérêt.

\section{Références}

1. Tenaillon O, Skurnik D, Picard B, Denamur E (2010) The population genetics of commensal Escherichia coli. Nat Rev Microbiol 8:207-17

2. Russo TA, Johnson JR (2003) Medical and economic impact of extraintestinal infections due to Escherichia coli: focus on an increasingly important endemic problem. Microbes Infect $5: 449-56$

3. Gordon DM, Clermont O, Tolley H, Denamur E (2008) Assigning Escherichia coli strains to phylogenetic groups: multilocus sequence typing versus the PCR triplex method. Environ Microbiol 10:2484-96

4. Picard B, Garcia JS, Gouriou S, et al (1999) The link between phylogeny and virulence in Escherichia coli extraintestinal infection. Infect Immun 67:546-53

5. Duriez P, Clermont O, Bonacorsi S, et al (2001) Commensal Escherichia coli isolates are phylogenetically distributed among geographically distinct human populations. Microbiology 147:1671-6

6. Johnson JR, Goullet P, Picard B, et al (1991) Association of carboxylesterase $\mathrm{B}$ electrophoretic pattern with presence and expression of urovirulence factor determinants and antimicrobial resistance among strains of Escherichia coli that cause urosepsis. Infect Immun 59:2311-5

7. Cooke NM, Smith SG, Kelleher M, Rogers TR (2010) Major differences exist in frequencies of virulence factors and multidrug resistance between community and nosocomial Escherichia coli bloodstream isolates. J Clin Microbiol 48:1099-104

8. Jaureguy F, Carbonnelle E, Bonacorsi S, et al (2007) Host and bacterial determinants of initial severity and outcome of Escherichia coli sepsis. Clin Microbiol Infect 13:854-62

9. Johnson JR, Kuskowski MA, Gajewski A, et al (2004) Virulence characteristics and phylogenetic background of multidrugresistant and antimicrobial-susceptible clinical isolates of Escherichia coli from across the United States, 2000-2001. J Infect Dis 190:1739-44

10. Vila J, Simon K, Ruiz J, et al (2002) Are quinolone-resistant uropathogenic Escherichia coli less virulent? J Infect Dis 186:1039-42

11. Horcajada JP, Soto S, Gajewski A, et al (2005) Quinoloneresistant uropathogenic Escherichia coli strains from phylogenetic group B2 have fewer virulence factors than their susceptible counterparts. J Clin Microbiol 43:2962-4

12. Skurnik D, Le Menac'h A, Zurakowski D, et al (2005) Integronassociated antibiotic resistance and phylogenetic grouping of Escherichia coli isolates from healthy subjects free of recent antibiotic exposure. Antimicrob Agents Chemother 49:3062-5

13. Hacker J, Kaper JB (2000) Pathogenicity islands and the evolution of microbes. Annu Rev Microbiol 54:641-79

14. Johnson JR, Kuskowski M (2000) Clonal origin, virulence factors, and virulence. Infect Immun 68:424-5
15. Johnson JR, Clermont O, Menard M, et al (2006) Experimental mouse lethality of Escherichia coli isolates, in relation to accessory traits, phylogenetic group, and ecological source. J Infect Dis 194:1141-50

16. Tourret J, Diard M, Garry L, et al (2010) Effects of single and multiple pathogenicity island deletions on uropathogenic Escherichia coli strain 536 intrinsic extra-intestinal virulence. Int J Med Microbiol 300:435-9

17. Soto SM, Jimenez de Anta MT, Vila J (2006) Quinolones induce partial or total loss of pathogenicity islands in uropathogenic Escherichia coli by SOS-dependent or -independent pathways, respectively. Antimicrob Agents Chemother 50:649-53

18. Johnson JR, Johnston B, Kuskowski MA, et al (2005) Spontaneous conversion to quinolone and fluoroquinolone resistance among wild-type Escherichia coli isolates in relation to phylogenetic background and virulence genotype. Antimicrob Agents Chemother 49:4739-44

19. Touchon M, Hoede C, Tenaillon O, et al (2009) Organised genome dynamics in the Escherichia coli species results in highly diverse adaptive paths. PLoS Genet 5:e1000344

20. Hendrickson H (2009) Order and disorder during Escherichia coli divergence. PLoS Genet 5:e1000335

21. Lescat M, Calteau A, Hoede C, et al (2009) A module located at a chromosomal integration hot spot is responsible for the multidrug resistance of a reference strain from Escherichia coli clonal group A. Antimicrob Agents Chemother 53:2283-8

22. Canton R, Coque TM (2006) The CTX-M beta-lactamase pandemic. Curr Opin Microbiol 9:466-75

23. Kumarasamy KK, Toleman MA, Walsh TR, et al (2010) Emergence of a new antibiotic resistance mechanism in India, Pakistan, and the UK: a molecular, biological, and epidemiological study. Lancet Infect Dis 10:597-602

24. Branger C, Zamfir O, Geoffroy S, et al (2005) Genetic background of Escherichia coli and extended-spectrum betalactamase type. Emerg Infect Dis 11:54-61

25. Nicolas-Chanoine MH, Blanco J, Leflon-Guibout V, et al (2008) Intercontinental emergence of Escherichia coli clone O25:H4ST131 producing CTX-M-15. J Antimicrob Chemother 61:273-81

26. Clermont O, Lavollay M, Vimont S, et al (2008) The CTXM-15-producing Escherichia coli diffusing clone belongs to a highly virulent B2 phylogenetic subgroup. J Antimicrob Chemother 61:1024-8

27. Peirano G, Schreckenberger PC, Pitout JD (2011) Characteristics of NDM-1-producing Escherichia coli isolates that belong to the successful and virulent clone ST131. Antimicrob Agents Chemother 55:2986-8

28. Lefort A, Panhard X, Clermont O, et al (2011) Host factors and portal of entry outweigh bacterial determinants to predict the severity of Escherichia coli bacteremia. J Clin Microbiol 49:777-83

29. Landraud L, Jauréguy F, Frapy E, et al (2011) Severity of Escherichia coli bacteraemia is independant of the intrinsic virulence of the strains assessed in a mouse model. Clin Microbiol Infect 2011:doi: 10.1111/j.1469-0691.2011.03750.x. [Epub ahead of print] 